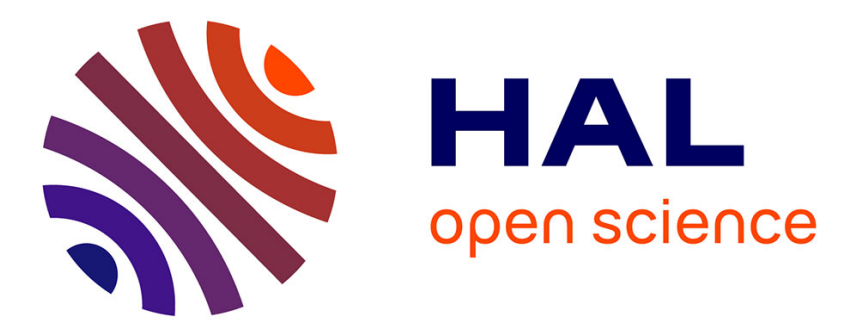

\title{
Site-Specified Magnetic States in Ferrites Probed by Magnetic Circular X-Ray Dichroism
}

\author{
N. Kawamura, H. Maruyama, K. Kobayashi, I. Inoue, H. Yamazaki
}

\section{To cite this version:}

N. Kawamura, H. Maruyama, K. Kobayashi, I. Inoue, H. Yamazaki. Site-Specified Magnetic States in Ferrites Probed by Magnetic Circular X-Ray Dichroism. Journal de Physique IV Proceedings, 1997, 07 (C1), pp.C1-269-C1-270. 10.1051/jp4:19971105 . jpa-00254726

\section{HAL Id: jpa-00254726 https://hal.science/jpa-00254726}

Submitted on 1 Jan 1997

HAL is a multi-disciplinary open access archive for the deposit and dissemination of scientific research documents, whether they are published or not. The documents may come from teaching and research institutions in France or abroad, or from public or private research centers.
L'archive ouverte pluridisciplinaire HAL, est destinée au dépôt et à la diffusion de documents scientifiques de niveau recherche, publiés ou non, émanant des établissements d'enseignement et de recherche français ou étrangers, des laboratoires publics ou privés. 


\title{
Site-Specified Magnetic States in Ferrites Probed by Magnetic Circular X-Ray Dichroism
}

\author{
N. Kawamura, H. Maruyama, K. Kobayashi, I. Inoue and H. Yamazaki \\ Department of Physics, Faculty of Science, Okayama University, 3-1-1 Tsushima-Naka, Okayama 700, \\ Japan
}

\begin{abstract}
To study magnetic states in ferrimagnetic Fe-oxides, we have measured magnetic circular $x$-ray dichroism at $K$-edge of $3 d$-transition metal. The observed dichroic signals are separately assigned to the contribution come from $3 d$-cation in tetrahedral or octahedral sites. We discuss the magnetic states of $3 d$-metal ion in terms of local enviroment.
\end{abstract}

\section{INTRODUCTION}

Magnetic circular X-ray dichroism (MCXD) is a powerful technique for studying magnetic states in magnetic materials because of an element-specificity, a shell-selectivity, and an angular momentum sensitivity. To clarify the difference in magnetic states of $3 d$-transition metal $(M)$ ions depending on local environment, e.g., tetrahedral $\left(T_{d}\right)$ or octahedral $\left(O_{h}\right)$ symmetry, we have applied this technique to various Fe-oxide ferrimagnets with the spinel or garnet structure. In general, $K$-edge absorption spectrum in M-oxides is characterized by a pre-peak structure and an enhanced main-peak one around the edge. The pre-peak has been assigned to the $1 s \rightarrow 3 d$ dipole-allowed transitions in $T_{d}$-sites whereas the main-peak is properly related to the $1 s \rightarrow 4 p$ transitions [1]. In this report we distinguish between the contributions of $T_{d}$ and $O_{h}$ sites to the spectrum through the preferred substitution of $\mathrm{M}$-ion in spinel and the difference in siteoccupation between spinel and garnet. Hence, the magnetic states of M-ion are discussed in terms of crystal field and local symmetry.

\section{EXPERIMENT}

The Fe-oxides studied in this work include $\mathrm{MFe}_{2} \mathrm{O}_{4}(\mathrm{M}=\mathrm{Mn}, \mathrm{Fe}, \mathrm{Co}$, and $\mathrm{Ni}), \gamma-\mathrm{Fe}_{2} \mathrm{O}_{3}$, and $\mathrm{LiFe}_{5} \mathrm{O}_{8}$ as spinel-type, $\mathrm{R}_{3} \mathrm{Fe}_{5} \mathrm{O}_{12}$ ( $\mathrm{R}=\mathrm{Y}$ and $\mathrm{Ho}$ ) as garnet-type, and $\mathrm{BaFe}_{12} \mathrm{O}_{19}$ as magnetoplumbite-type. MCXD spectrum was recorded in transmission mode for powder sample using the left-circularly polarized x-rays on BL-28B at Photon Factory 2.5 $\mathrm{GeV}$ storage-ring in KEK. The MCXD spectrum $(\Delta \mu t)$ is defined as the difference in absorption with reversing the direction of magnetic field: $\Delta \mu t=[\mu t(\hat{\imath})-\mu t(\downarrow)]$, and $\mathrm{x}$-ray absorption near-edge structure (XANES : $\mu t)$ is shown as the average: $\mu t=[\mu t(\uparrow)+\mu t(\downarrow)] / 2$, where $\uparrow(\downarrow)$ represents the antiparallel (parallel) configuration of magnetic field with respect to the wave vector of incident X-ray. The MCXD spectrum was systematically measured at the $K$-edge of $\mathrm{Mn}, \mathrm{Fe}, \mathrm{Co}$, and $\mathrm{Ni}$.

\section{RESULTS AND DISCUSSION}

Fe $K$-edge XANES and MCXD spectra are shown in Figures 1(a) and 1(b), respectively. In the case of inverse spinel, the substitution of $\mathrm{Co}^{2+}, \mathrm{Ni}^{2+}, \mathrm{Li}^{+}$ion, or defect for $\mathrm{Fe}^{2+}$ in $\mathrm{O}_{h}$-sites causes scarcely any change in the XANES spectrum; on the other hand, in the case of $\mathrm{Mn}$-ferrite with the normal spinel structure, the substitution of $\mathrm{Mn}^{2+}$ ion for $\mathrm{Fe}^{3+}$ in $T_{d}$-sites induces a significant reduction of pre-peak intensity. On the contrary, in the garnet-type ferrite including more $T_{d}$-sites, the pre-peak intensity clearly increases in comparison with that in spinel. These observations are in good agreement with the assignment that the pre-peak is attributed to the cations in $T_{d}$-sites. It is not clear, however, whether the main-peak can be associated with the $O_{h}$-sites or not, because its feature is spread over the large continuum background resulting from the $1 s \rightarrow 4 p$ dipole transitions.

To clarify this, magnetic effect in XANES spectrum, i.e., dichroic signal, will be effectual, because it provides the information on magnetic polarization in the interesting electronic states. Figure $1(b)$ shows the Fe $K$-edge MCXD in this series. The observed spectrum is formed a remarkable dispersion-type profle and located in both the pre-peak $(\sim 0 \mathrm{eV})$ and the main-peak $(\sim 15 \mathrm{eV})$ regions. In Mn-ferrite, the dichroism almost disappears in the pre-peak region but yields prominent spectrum at the main-peak position. In the other ferrites, the intensity ratio of MCXD at main-peak to that at pre-peak is consistent with the substitution for the Fe ion in $O_{h}$-sites. In garnet ferrite, the dispersion-type dichroic signal is clealy observed only in the pre-peak region and shows an opposite sign to that in spinel, which is interpreted as the magnetic site dominantly contributed to bulk magnetization in ferrimagnet. Harada and Kotani [2] have theoretically reproduced the dispersion-type dichroic spectrum of $\mathrm{Fe}^{3+}$ ion in the $T_{d}-$ or $O_{h}$-symmetry by taking $p-d$ hybridization with $O^{2-}$ ligands and spin-orbit coupling in $t_{2 g}$ orbitals into account. We 
can associate the dichroism in the main-peak region with Fe ion in $O_{h}$-sites. However, a subtle MCXD signal in the main-peak region in garnet is still an open question.

To verify our assignment, moreover, we have measured XANES and MCXD spectra concerning the substituted $\mathrm{M}$-ion in spinel. Figures 2(a) and 2(b) show the Co and $\mathrm{Ni} K$-edge spectra, respectively. In XANES, the spectra exhibit an enhanced white-line like absorption and a substantial reduction of pre-peak intensity. In MCXD, a positive-doublepeak appears at the position corresponding to the white-line like absorption, and no dichroic signal at the pre-peak is observed. These experimental facts demonstrate that the magnetic $\mathrm{Co}^{2+}$ or $\mathrm{Ni}^{2+}$ ion is preferably substituted for the $\mathrm{Fe}^{2+}$ ion in $O_{h}$-sites. The similarity between the Co and Ni MCXD spectra may be related to a resemblance of local magnetic states in such divalent ion in spite of the difference in the ground-state 3d-electronic configurations. Although there is no interpretation of such positive-double-peak dichroic spectrum, it is associated with crystal-field splitting, exchange interaction, charge-transfer, $p-d$ hybridization, etc. For the $\mathrm{Mn} K$-edge, the characteristic dispersion-type spectrum is expected because of the same $3 d^{5}$ electronic configuration as the Fe ${ }^{3+}$ ion. Actually, such dichroic signal is observed in a wide region of the edge, so that the structure is very complicated and possibly related to the inverse spinel structure. Consequently, we conclude that the dichroic signal in the main-peak region is reasonably attributed to $\mathrm{Fe}$ ion in $O_{h}$-sites.

Let us briefly discuss the magnetic states of $\mathrm{Fe}^{3+}$ ion in terms of crystal field and local symmetry. The dichroism can be interpreted as the mechanism [2] composed of the dipole-allowed $1 s \rightarrow 3 d$ transitions, which is due to the hybridization between Fe $3 d$ and ligand $2 p$ orbitals, and the spin-orbit coupling in the $3 d-s t a t e s$, which leads to a splitting in the degenerated $t_{2 g}$ and $e_{g}$ multiplets. Hence, the MCXD is very sensitive to the local enviroment of polyhedron. The ground state electronic configurations of the cation in $O_{h}$-symmetry studied in this work are in a high-spin state, so that the dichroism for the substituted cations range from $\mathrm{Mn}\left(3 d^{5}\right)$ to $\mathrm{Ni}\left(3 d^{8}\right)$ is essentially related to the electron-filling of the $3 d t_{2 g}$-states.

(a)

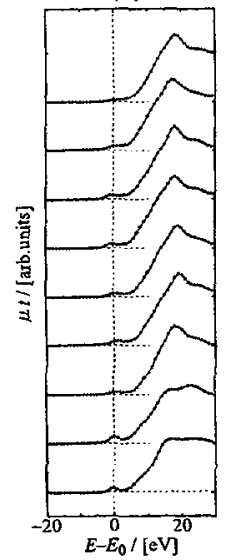

(b)

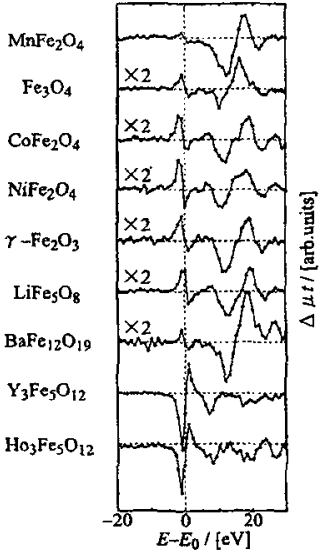

Figure 1: (a) XANES and (b) MCXD spectrum at the Fe $K$-edge. Absorption edge energy $E_{0}$ was determined as the position of the pre-peak structure, which is constant regardless of the samples within the experimental error.

(a)

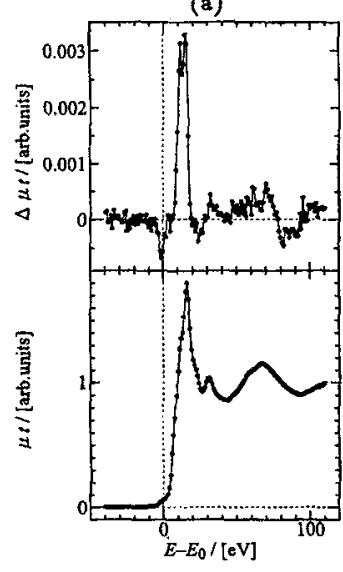

(b)

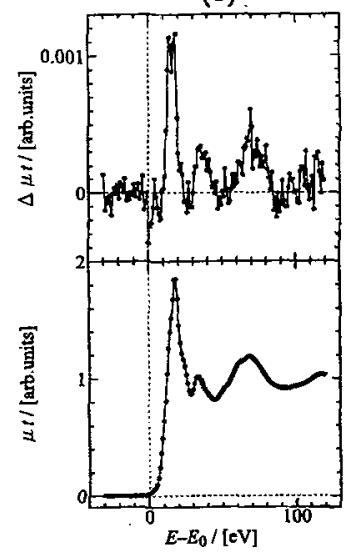

Figure 2: XANES (lower panel) and MCXD (upper panel) spectra (a) at the Co $K$-edge in Co in Co-ferrite and (b) at the $\mathrm{Ni} K$-edge in Ni-ferrite.

\section{CONCLUSION}

We have measured the XANES and MCXD spectra at $K$-edge of $\mathrm{Mn}, \mathrm{Fe}, \mathrm{Co}$, and $\mathrm{Ni}$ in the various ferrimagnetic oxides with the spinel or garnet structure. For the Fe $K$-edge spectra, we have verified that the pre-peak (main-peak) structure is attributed to the cations in $T_{d}$ (mainly $O_{h}$ ) sites, through the preferred substitution in spinel and the difference in site-occupation between spinel and garnet. Since the MCXD is very sensitive to the local environment of polyhedron, it is useful for studying the magnetic states of $3 d$-ions in a local symmetry.

\section{Acknowledgments}

The authors wish to acknowledge stimulating discussions and suggestions from Prof. I.Harada of Okayama University. This work has been performed with the approval of the Photon Factory Program Advisory Committee(Proposal No.92-G185).

\section{References}

[1] L.A.Grunes, Phys.Rev.B27(1983) 2111-2131.

[2] I.Harada and A.Kotani, J.Phys.Soc.Jpn.63(1994) 1285-1288. 